\title{
Editorial
}

\section{Does an impaired flow mediated vasodilatation predict hypertension in offspring hypertensive parents?}

The vascular endothelium is an essential organ that exerts important roles in the cardiovascular system. Not only does it control underlying smooth muscle tone, but it also modulates other functions such as activation of leucocytes and platelets, the coagulation cascade, vascular permeability, and proliferation of vascular smooth muscle cells. Vascular smooth muscle tone is continuously regulated by a delicate balance of vasodilating (that is, nitric oxide (NO), prostacyclin, and endothelium derived hyperpolarising factor) and contracting (that is, endothelin-1, thromboxane $\mathrm{A} 2$, prostaglandin $\mathrm{H} 2$, and superoxide anion) substances.

Dysfunction of the endothelium is generally expressed by either a decreased release of these vasodilators, or increased production of endothelium derived vasoconstrictors, or both.

Human essential hypertension has been associated with alterations in endothelial function. Indeed, most studies have shown blunted forearm and coronary blood flow responses to muscarinic agonists, along with a preserved response to sodium nitroprusside. Furthermore, inhibition of NO synthase has suggested that basal NO mediated vasodilatation is abnormal in patients with essential hypertension. In addition, measurement of a specific biochemical marker of the L-arginine-NO pathway-conversion of $\mathrm{L}-\left[{ }^{15} \mathrm{~N}\right]_{2}$-arginine to $\left[{ }^{15} \mathrm{~N}\right]$ nitrate- has shown that the basal production of NO is decreased in essential hypertension. Flow mediated vasodilatation is a useful index of endothelial function. ${ }^{1}$ It measures the change in the calibre of conductance arteries (that is, brachial, radial or femoral artery) during reactive hyperaemia, a manoeuvre that increases blood flow (shear stress) through the vessels. This response is compared with the vasodilatation produced by the sublingual administration of nitroglycerin, an index of endothelium independent vasodilatation. Previous reports have suggested that in human essential hypertension, endothelial function is also impaired in large arteries. ${ }^{23}$ However, this impaired endothelial response is not a universal finding. ${ }^{4}$

Although most of the present evidence in human essential hypertension suggests an altered endothelial function, it is still unclear whether this defect is a cause or effect of increased blood pressure. Some studies have hypothesised a relation between polymorphism in the endothelial NOS gene and human essential hypertension. ${ }^{5}$ Other reports using invasive techniques have shown a reduction in acetylcholine induced vasodilatation, ${ }^{6}$ and a blunted forearm vasoconstrictor response to $\mathrm{L}-\mathrm{N}$ monomethyl arginine (L-NMMA) in offspring of hypertensive parents ${ }^{7}$. Furthermore, Noll and colleagues have demonstrated an enhanced plasma endothelin response to mental stress in normotensive offspring of hypertensive parents. ${ }^{8}$ Taken together, these results suggest that endothelial dysfunction precedes the onset of essential human hypertension. On the other hand, there are also studies that suggest the converse. For instance, Millgard and Lind showed that an acute increase in blood pressure induced by noradrenaline (norepinephrine) resulted in impaired endothelium dependent vasodilatation in healthy volunteers. ${ }^{9}$ Furthermore, normalisation of blood pressure with different groups of antihypertensive drugs restored the vasoconstrictor response to L-NMMA in patients with essential hypertension. ${ }^{10}$

\section{Endothelial dysfunction, essential hypertension, and insulin}

The relation between endothelial dysfunction, essential hypertension, and insulin resistance is still unclear. Insulin resistance is characterised by decreased rates of insulin mediated glucose uptake, and it is usually associated with systemic hypertension, dyslipidaemia, and non-insulin dependent diabetes mellitus. Both insulin resistance and essential hypertension have been linked with endothelium dysfunction and an altered insulin mediated vasodilatation. ${ }^{11}$ Insulin is an arterial vasodilator in skeletal muscle vascular beds, and there is evidence that endothelial NO mediates this vascular effect. ${ }^{12}$ Petrie and colleagues have shown a positive relation between insulin sensitivity and forearm vasoconstrictor responses to L-NMMA in healthy volunteers. ${ }^{13}$ However, as yet it has not been determined what the role of endothelial dysfunction and insulin resistance is in the pathogenesis of essential human hypertension.

In this issue of Heart, Žižek and colleagues report that a large population of essential hypertensive patients had an abnormal flow-dependent vasodilatation in response to reactive hyperaemia. ${ }^{14}$ This confirms previous findings of an impaired endothelium dependent vasodilatation in the brachial artery of patients with essential hypertension. ${ }^{23}$ An interesting observation in this finding, however, is that the nitroglycerin (glyceryl trinitrate) induced vasodilatation was impaired, suggesting that the functional integrity of cyclic GMP dependent vasodilatation in the vascular smooth muscle may also be affected. This is in divergence with most previous endothelial function studies performed in essential hypertensive patients, in which the endothelium independent vasodilatation to $\mathrm{NO}$ releasing substances was preserved. This finding highlights the concept that an abnormal flow mediated vasodilatation in this pathology may be suggestive of a multifactorial process involving not only a general endothelial dysfunction, but also an altered vascular smooth muscle cell function.

\section{Clinical implications}

The potential clinical implications of this result could be related to the progress of arteriosclerosis, the low efficacy of organic nitrates in patients with concomitant coronary artery disease (if the brachial arteries reflect the behaviour of coronary arteries), and the incidence of anginal attacks during aerobic exercise in essential hypertensive patients. A major limitation of this study, however, is that endothelial function in the patient group was not assessed at baseline (off treatment), and therefore it cannot be rule out an 
improvement on endothelial function was produced by the antihypertensive drugs.

Žižek and colleagues have also shown that flow mediated vasodilatation was significantly lower in normotensive young subjects who had a family history of essential hypertension compared with offspring of normotensive parents. ${ }^{14}$ This result is consistent with the hypothesis that endothelial dysfunction is a cause rather than a consequence of hypertension. However, we have to be cautious with the interpretation of this finding since parameters such as glucose, triglycerides, and plasma insulin-which alter endothelial responsiveness-were higher in offspring of hypertensive parents than those in the control group. Body mass index was also another predictor of endothelial dysfunction in this study. Since offspring of hypertensive parents had a significantly higher body mass index than those in the control group, it is likely that this variable may have confounded the association between familiar predisposition to hypertension and endothelial dysfunction. However, arterial blood pressure, which was still within the normal range, was significantly higher in the offspring of essential hypertensive parents than in the control group. Although it is not definite whether this group of offspring of hypertensive parents will become hypertensive, the data show a tendency toward raised blood pressure even at young age.

The authors reported that the offspring of hypertensive parents also had higher plasma insulin concentrations compared with the offspring of normotensive parents. In addition, plasma insulin concentration was a "borderline" predictor of endothelial dysfunction in this study. Significant limitations are, however, that insulin resistance was not evaluated in this study and body mass index, which is recognised as a strong predictor of insulin resistance, could have acted as a confounding factor in this association.

In conclusion, this study suggests that an altered flow dependent vasodilatation in offspring of hypertensive parents may precede the onset of essential hypertension. However, this results need to be confirmed in a long term prospective study, including a younger population of offspring of essential hypertensive parents who are well matched for confounding factors that influence both endothelial function and insulin sensitivity.

PABLO FORTE NIGEL BENJAMIN

Department of Clinical Pharmacology,

St Bartholomew's and the Royal London School of Medicine and

Dentistry,

Charterhouse Square,

London EC1M 6BQ, UK

p.e.forte@mds.qmw.ac.uk

n.benjamin@qmw.ac.uk

1 Celermajer DS, Sorensen KE, Gooch VM, et al. Non-invasive detection of endothelial dysfunction in children and adults at risk of atherosclerosis. endothelial dysfunction in

2 Iiyama K, Nagano M, Yo Y, et al. Impaired endothelial function with essential hypertension assessed by ultrasonography. Am Heart f 1996;132:77982.

3 Muiesan ML, Salvetti M, Monteduro C, et al. Effect of treatment on flowdependent vasodilation of the brachial artery in essential hypertension. Hypertension 1999;33:575-80.

4 Laurent S, Lacolley P, Brunel P, et al. Flow-dependent vasodilation of brachial artery in essential hypertension. Am F Physiol 1990;258:H1004-11.

5 Miyamoto Y, Saito Y, Kajiyama N, et al. Endothelial nitric oxide synthase gene is positively associated with essential hypertension. Hypertension 1998; 32:3-8.

6 Taddei S, Virdis A, Mattei P, et al. Defective L-arginine-nitric oxide pathway in offspring of essential hypertensive patients. Circulation 1996;94:1298.

7 Mcallister AS, Atkinson AB, Johnston GD, et al. Basal nitric oxide production is impaired in offspring of patients with essential hypertension Clin Sci tion is impaired in

8 Noll G, Wenzel RR, Schneider M, et al. Increased activation of sympathetic Noll G, Wenzel RR, Schneider M, et al. Increased activation of sympathetic
nervous system and endothelin by mental stress in normotensive offspring of hypertensive parents. Circulation 1996;93:866-9.

9 Millgård J, Lind L Acute hypertension impairs endothelium-dependent vasodilatation. Clin Sci 1998;94:601-7.

10 Lyons D, Webster J, Benjamin N. The effect of antihypertensive therapy on local intra-arterial $\mathrm{N}^{\mathrm{G}}$-monomethyl-L-arginine in patients with essential hypertension. F Hypertens 1994;12:1047-52.

11 Baron AD, Steinberg HO. Endothelial dysfunction, insulin sensitivity, and hypertension. Circulation 1997;96:725-6.

12 Scherrer U, Randin D, Vollenweider P, et al. Nitric oxide release accounts for insulin's vascular effects in humans. $\mathcal{F}$ Clin Invest 1994;94:2511-15.

13 Petrie JR, Ueda S, Webb DJ, et al. Endothelial nitric oxide production and insulin sensitivity. A physiological link with implications for pathogenesis of insulin sensitivity. A physiological link with implication

14 Žižek B, Poredos P, Videcnic B. Endothelial dyfunction in hypertensive patients in normotensive offspring on subjects with essential hypertension. Heart 2001;85:215-6.

\section{Commentary by the Editor (see p133)}

In this issue of Heart we publish the guidelines on the management of unstable angina, which have been written jointly by the Royal College of Physicians and the British Cardiac Society. ${ }^{1}$ They follow quite closely on the heels of the US guidelines on the same subject. ${ }^{2}$ However, they take a somewhat different approach. The US guidelines are long (nearly 100 pages), exhaustive, and rather cumbersome, although very informative. The British guidelines are much briefer (10 pages), more user friendly, and to the point. Both will be useful to the physician and cardiologist, but the British ones are better value for the time expended reading them!

Another set of guidelines, however useful and informative, raises the issue of the place and authority of guidelines. They should be exactly what they say they are-guidance. However, increasingly in the current nervous medical atmosphere, their observance is being interpreted as mandatory. The quality of medicine, and therefore patient care, will suffer if treatment is based only upon guidelines, and these guidelines are not interpreted and adjusted in the light of the physician's experience of both the condition and the patient. Latitude to deviate thoughtfully from the guidelines must be preserved. It is particularly important since the so called "evidence base" upon which such "evidence based" guidelines are produced is frequently flawed and/or incomplete when examined closely.

The potential danger and misuse of guidelines is emphasised by the very real possibility that commercial companies, whose products are recommended by the guidelines, will use their own interpretation of the guidelines to pressurise physicians into using their products.

Thus, although guidelines are extremely important, they must remain guidelines otherwise medicine will sink into a state where it is practised by rote and not by intelligent thought.

ROGER HALL

1 British Cardiac Society Guidelines and Medical Practice Commitee, and Royal College of Physicians Clinical Effectiveness and Evaluation Unit. Guideline for the management of patients with acute coronary syndromes without persistent ECG ST segment elevation. Heart 2001;85:133-42.

2 American College of Cardiology/American Heart Association Task Force on Practice Guidelines (Committee on the Management of Patients with Unstable Angina). ACC/AHA guidelines for the management of patients with unstable angina and non-ST-segment elevation myocardial infarction. $\mathcal{f}$ Am Coll Cardiol 2000;36: $970-1062$ 